\title{
Vascular improvements in individuals with type 2 diabetes following a 1 year randomised controlled exercise intervention, irrespective of changes in cardiorespiratory fitness
}

\author{
Megan Hetherington-Rauth ${ }^{1} \cdot$ João P. Magalhães ${ }^{1} \cdot$ Pedro B. Júdice $^{1} \cdot$ Xavier Melo $^{1,2} \cdot$ Luís B. Sardinha $^{1}$ (D
}

Received: 7 September 2019 / Accepted: 18 December 2019 /Published online: 20 January 2020

(C) Springer-Verlag GmbH Germany, part of Springer Nature 2020

\begin{abstract}
Aims/hypothesis Vascular changes in individuals with type 2 diabetes mellitus majorly contribute to the development of cardiovascular disease. Increased cardiorespiratory fitness (CRF) has been associated with improvements in vascular health. Although CRF tends to improve with exercise training, there remains a portion of participants with little or no improvement. Given the importance of vascular function in individuals with type 2 diabetes, we assessed whether individuals who failed to improve CRF following a 1 year exercise intervention also failed to improve arterial stiffness and structural indices.

Methods Individuals with type 2 diabetes with no major micro- and macrovascular complications and aged between 30 and 75 years old $(n=63)$ participated in a three-arm, 1 year, randomised controlled exercise intervention in Lisbon, Portugal. The study involved a non-exercise control group, a moderate continuous training combined with resistance training (RT) group and a high-intensity interval training with RT group. Allocation of participants into the intervention and control groups was done using a computer-generated list of random numbers. An improvement in CRF was defined as a change in $\dot{V} \mathrm{O}_{2 \text { peak }} \geq 5 \%$. Vascular stiffness and structural indices were measured using ultrasound imaging and applanation tonometry. Generalised estimating equations were used to compare changes in vascular measures across individuals in the control group $(n=22)$ and those in the exercise groups who either had improved CRF (CRF responders; $n=14$ ) or whose CRF did not improve (CRF non-responders; $n=27$ ) following 1 year of exercise training.

Results Compared with the control group, exercisers, with and without improvements in CRF, had decreased carotid intimamedia thickness (IMT) (CRF responders: $\beta=-2.84$ [95\% CI $-5.63,-0.04]$; CRF non-responders: $\beta=-5.89$ [95\% CI -9.38 , $-2.40]$ ) and lower-limb pulse wave velocity (PWV) (CRF responders: $\beta=-0.14$ [95\% CI $-0.25,-0.03]$; CRF non-responders: $\beta=-0.14[95 \% \mathrm{CI}-0.25,-0.03])$, the latter being an indicator of peripheral arterial stiffness. Only CRF responders had decreased PWV of the upper limb compared with control participants $(\beta=-0.12[95 \% \mathrm{CI}-0.23,-0.01])$. As for central stiffness, CRF non-responders had increased aortic PWV compared with CRF responders $(\beta=0.19$ [95\% CI 0.07, 0.31]), whereas only the CRF non-responders had altered carotid distensibility coefficient compared with the control group $(\beta=0.00[95 \%$ CI $3.01 \times$ $\left.\left.10^{-5}, 0.00\right]\right)$. No interaction effects between the CRF responders and non-responders vs control group were found for the remaining stiffness or haemodynamic indices $(p>0.05)$.
\end{abstract}

Electronic supplementary material The online version of this article (https://doi.org/10.1007/s00125-020-05089-5) contains peer-reviewed but unedited supplementary material, which is available to authorised users.

Luís B. Sardinha

1sardinha@fmh.ulisboa.pt

1 Exercise and Health Laboratory, CIPER, Faculdade de Motricidade Humana, Universidade de Lisboa, Estrada da Costa, 1499-002 Cruz-Quebrada, Portugal

2 Ginásio Clube Português, GCP Lab, Lisbon, Portugal 


\section{Research in context}

\section{What is already known about this subject?}

- Adverse vascular changes are thought to be a major mechanism connecting diabetes with increased risk of cardiovascular disease (CVD)-associated morbidity and mortality

- Increased cardiorespiratory fitness (CRF) following exercise training is highly related to improvements in functional and structural arterial properties

- Considerable interindividual differences exist in the amount of change in CRF following exercise, with a substantial number of adults showing no improvement in CRF

\section{What is the key question?}

- Do individuals with type 2 diabetes who lack improvements in CRF following a 1 year exercise intervention also fail to improve arterial stiffness and structural indices?

\section{What are the new findings?}

- Regardless of improvements in CRF, individuals with type 2 diabetes had significant improvements in arterial structure and stiffness indices following 1 year of exercise training

\section{How might this impact on clinical practice in the foreseeable future?}

- The impact of exercise training on vascular health indicators should not be overlooked by simply focusing on CRF since vascular-health benefits are still obtainable with exercise in individuals with type 2 diabetes despite lack of improvements in fitness

Conclusions/interpretation Regardless of improvements in CRF, individuals with type 2 diabetes had significant improvements in carotid IMT and lower-limb arterial stiffness following a 1 year exercise intervention. Thus, a lack of improvement in CRF following exercise in people with type 2 diabetes does not necessarily entail a lack of improvement in vascular health.

Trial registration ClinicalTrials.gov NCT03144505

Funding This work was supported by fellowships from the Portuguese Foundation for Science and Technology. This work is also financed by a national grant through the Fundação para a Ciência e Tecnologia (FCT), within the unit I\&D 472.

Keywords Arterial stiffness $\cdot$ Carotid intima-media thickness $\cdot$ Exercise intervention $\cdot$ Peak wave velocity $\cdot$ Type 2 diabetes

\section{Abbreviations}

CRF Cardiorespiratory fitness

CVD Cardiovascular disease

DBP Diastolic BP

HIIT High-intensity interval training

HRR Heart rate reserve

IMT Intima-media thickness

LIPA Low intensity physical activity

MAP Mean arterial pressure

MCT Moderate continuous training

MVPA Moderate-to-vigorous physical activity

NEPA Non-exercise physical activity

PWV Pulse wave velocity

RT Resistance training

SBP Systolic BP

TEM Technical error of measurement

\section{Introduction}

Alterations in arterial structure and stiffness are major culprits for the increased risk of cardiovascular disease (CVD) and early mortality in individuals with type 2 diabetes $[1,2]$. Pulse wave velocity (PWV) and carotid intima-media thickness (IMT) are established surrogate measures of arterial stiffness and structure, respectively, that are strong predictors of cardiovascular outcomes [2-4]. Hence, interventions in individuals with type 2 diabetes that can improve these markers of arterial health are critically important for preventing and/or delaying adverse cardiovascular events and mortality. Exercise training of different types and intensities has been shown to be an effective strategy for improving PWV and IMT in individuals with type 2 diabetes [5-8]. 
Many of the cardiovascular benefits of exercise are associated with improvements in cardiorespiratory fitness (CRF), as determined by $\dot{V} \mathrm{O}_{2 \text { peak }}$, independent of traditional risk factors [9]. A higher CRF is not only associated with lower levels of risk factors for the metabolic syndrome, lower body fat, improved insulin resistance and BP, and reduced inflammation [10], all of which are independently associated with arterial structure and stiffness [11], but it is also independently associated with reduced carotid IMT [12] and increased PWV in individuals with and/or at risk for type 2 diabetes [13]. However, there is still wide interindividual variability in the response to exercise, with certain individuals improving CRF with exercise training, while others do not $[14,15]$. In fact, the theme of CRF response to exercise was recently highlighted in a consensus statement by an expert panel; the authors recommended future research to better understand the interindividual variability in the therapeutic effect of exercise [15].

Although CRF, based on $\dot{V} \mathrm{O}_{2}$, is connected with many health outcomes [10], previous investigations have suggested that exercise and being physically active can have beneficial effects on health that are independent of improvements in $\mathrm{CRF}$, in both healthy individuals [16], and those with increased cardiovascular risk $[17,18]$. However, in individuals with type 2 diabetes, there has only been one investigation that has addressed the impact of CRF response on health outcomes (the Health Benefits of Aerobic and Resistance Training in individuals with Type 2 Diabetes [HART-D] study), in which improvements in glycaemic control and body fat were observed regardless of improvements in CRF following a 9 month exercise intervention [14]. It is particularly important to consider the possibility of having health benefits from exercise despite alterations in CRF when working with individuals with type 2 diabetes as they are more likely not to experience improvements in CRF following exercise due to having slowed oxygen uptake, impaired muscle oxygen delivery, premature muscle fatigue and reduced overall exercise tolerance as a consequence of the disease [19]. No investigation, however, has assessed the long-term relationship between CRF non-response and measures of vascular stiffness and structure in individuals with type 2 diabetes. Given that adverse vascular changes are thought to be the major mechanism connecting diabetes with increased risk of CVD morbidity and mortality [2], and that functional and structural arterial properties are highly related to CRF $[12,13]$, it is uncertain whether exercise-induced vascular changes only occur in those who also have improved CRF. Therefore, we investigated arterial stiffness and structure indices in individuals with type 2 diabetes who either improved or did not improve CRF following a 1 year exercise intervention. We hypothesised that, following 1 year of exercise training, participants with type 2 diabetes would have improvements in arterial stiffness and structure irrespective of improvements in CRF, as compared with a non-exercise control group.

\section{Methods}

Study design and participants The current investigation assessed individuals with type 2 diabetes who took part in the D2FIT study. The design and methodology of the D2FIT study is fully described elsewhere [20]. In brief, the D2FIT study was a 1 year exercise randomised controlled trial with three distinct arms: a non-exercise control group, a moderate continuous training (MCT) with resistance training (RT) group, and a high-intensity interval training (HIIT) with RT group (ClinicalTrials.gov registration no. NCT03144505). The three groups were allocated with a 1:1:1 ratio by a researcher external to the project, using a computergenerated list of random numbers. The primary outcome of this study concerned changes in $\mathrm{HbA}_{1 \mathrm{c}}$ following exercise in individuals with type 2 diabetes, with secondary outcomes focused on several health-related parameters, including CRF and vascular health changes, which were assessed at baseline and at follow-up (1 year). Inclusion criteria consisted of the following: adults previously diagnosed with type 2 diabetes in accordance with ADA criteria [21]; aged between 30-75 years old; no major microvascular complications from diabetes, such as nephropathy, neuropathy and retinopathy, when considered severe enough to be contraindicative to exercise, as assessed by a clinical physician; no macrovascular complications, such as coronary artery disease and cerebral vascular disease; BMI $<48 \mathrm{~kg} / \mathrm{m}^{2}$; and no physical limitations that would prevent individuals from practicing exercise. Participants were recruited within the Lisbon Metropolitan Area (Portugal) between February 2014 and July 2016. Power and sample size calculations (G-Power, Version 3.1.3; Düsseldorf, Germany) were based on a predicted change in $\mathrm{HbA}_{1 \mathrm{c}}$ of 0.66 units with an SD of 1.2 units, $\alpha=0.05,1-\beta=0.80$, and a $10 \%$ expected dropout rate. For the D2FIT project, a total of 80 individuals were selected and randomised using a computergenerated list of random numbers. However, for this specific analysis, we considered only those who completed the investigation, resulting in a final sample size of 63 participants with type 2 diabetes.

Written informed consent was obtained from all participants prior to screening. The study protocol was reviewed and approved by the Ethics Committee of the Portuguese Diabetes Association (approval number: 07/17/2013).

Exercise intervention Exercise prescription for both exercise groups was standardised, according to body weight and based on physical activity guidelines [22], to achieve a weekly target of $41.84 \mathrm{~kJ} / \mathrm{kg}$ (10 kcal/kg) for both exercise groups. 
Participants in the HIIT with RT $(n=19)$ and MCT with RT groups $(n=22)$ exercised 3 days per week. Throughout the whole year of the intervention, individuals in both groups participated in a planned periodisation exercise prescription programme with individualised intensity based upon heart rate reserve (HRR), to gradually introduce exercise prescription. A heart rate Polar band (Polar T-31; Bethpage, NY, USA) was worn by participants during the exercise sessions and was used to monitor the intensity of training. The MCT group performed continuous cycling (Monark Ergometric 828e (Vansbro, Sweden) at $40-60 \%$ of HRR throughout the 1 year intervention. The HIIT group performed 1 min of cycling at $90 \%$ of HRR, followed by a $1 \mathrm{~min}$ rest period at $40-60 \%$ of HRR, using a 1:1 exercise:rest ratio. Following the aerobic exercise component, both groups underwent whole-body RT, which included one set of 10-12 repetitions of upper- and lowerlimb exercises. All exercise sessions were overseen and supervised by a certified exercise physiologist. A full detailed description of the periodisation protocol can be found elsewhere [20].

A standard counselling session regarding general physical activity guidelines was provided for the control group $(n=22)$. Additionally, the control group, along with both exercise groups, met once every 4 weeks for thematic sessions, with topics including nutrition or physical activity, to improve trial adherence.

Anthropometry Participants were weighed without shoes and wearing minimal clothing, on an electronic scale (Seca, Hamburg, Germany), to the nearest $0.01 \mathrm{~kg}$. Height was measured to the nearest $0.1 \mathrm{~cm}$ with a stadiometer (Seca), according to standardised procedures [23]. BMI $\left(\mathrm{kg} / \mathrm{m}^{2}\right)$ was calculated as the ratio between weight $(\mathrm{kg})$ and the square of height (m).

Brachial BP After $15 \mathrm{~min}$ in the supine position, the right and left brachial systolic BP (SBP) and diastolic BP (DBP) were measured using an automated oscillometric cuff (HEM-907E; Omron, Tokyo, Japan). Two measurements were taken, with a third measurement being performed if duplicate values deviated by $>5 \mathrm{mmHg}$. The mean arterial pressure (MAP) was calculated using the formula: $\mathrm{MAP}=\mathrm{DBP}+[1 / 3$ (SBP $\mathrm{DBP})]$. Pulse pressure was calculated as follows: pulse pressure $=\mathrm{SBP}-\mathrm{DBP}$.

Carotid artery IMT Carotid artery IMT was defined as the distance between the leading edge of the lumen-intima interface and the leading edge of the media-adventitia interface of the far wall of the right carotid artery. This measurement was performed using an ultrasound scanner equipped with a linear $13 \mathrm{MHz}$ probe (MyLab One, Esaote, Italy) [24] and distension curves were acquired within a segment of the carotid artery about $1 \mathrm{~cm}$ before the flow divider.
Carotid arterial stiffness indices After $15 \mathrm{~min}$ in a supine position, the carotid arterial stiffness measurement was conducted on the right side of the body. An ultrasound scanner equipped with a linear $13 \mathrm{MHz}$ probe (MyLab One), with Quality Arterial Stiffness technology, was used approximately $1 \mathrm{~cm}$ before the bifurcation. This allows the calculation of carotid stiffness indices: PWV $(\mathrm{m} / \mathrm{s})$, carotid distensibility coefficient $(1 / \mathrm{KPa})$ and stiffness index $\beta$. A detailed description of the calculations for the carotid stiffness indices can be found in the electronic supplementary material (ESM) Methods.

Contralateral PWV PWV was measured by applanation tonometry immediately after ultrasound imaging. A single operator located the carotid, femoral, radial and distal posterior tibial arteries on the left side of the body and marked the point for capturing the corresponding pressure curves with two specific pressure sensitive transducers. The distance between the carotid and femoral, and radial and distal posterior tibial arteries were measured and values were directly inserted into the Complior Analyse software (ALAM Medical, Paris, France). Detailed description of the methods for contralateral PWV are available in the ESM Methods. PWV values obtained from measurements of the carotid to femoral artery, carotid to radial artery, and carotid to distal posterior tibial artery were taken as indices of aortic and peripheral arterial stiffness for upper and lower limbs, respectively. The quality of the PWV records was immediately evaluated by a second observer. Whenever a continuous decrease before the sharp systolic upstroke was not clearly seen, or the tolerance was above $5 \mathrm{~m} / \mathrm{s}$, a second measure was taken.

Carotid BP Carotid SBP was obtained from left carotid traces acquired during the PWV assessment, from the Complior Analyser (ALAM Medical). The waveforms were averaged and the mean values were extracted from $15 \mathrm{~s}$ window of acquisition. The carotid waveforms were calibrated with left brachial MAP measured immediately before the acquisition.

CRF For the assessment of CRF, a Bruce standard protocol [25] was used on a motorised treadmill (Quinton, model Q-65; Cardiac Science, Bothell, WA, USA) to exhaustion, in a spacious ventilated room, with only the participant, the technician and the cardiologist present during the test. All graded exercise tests were monitored using a 12-lead ECG PC-based acquisition module (model Quark C12; Cosmed, Rome, Italy) and all data, including heart rate, were monitored and recorded using Cosmed software. Expired gases were continuously analysed, breath-by-breath, through a portable gas analyser (K4b2; Cosmed). To ensure peak effort, participants attained volitional fatigue and met at least one of the following objective test criteria: (1) a respiratory exchange ratio of 1.1 or higher; (2) participants reached predicted maximal heart rate; (3) oxygen uptake did not increase in spite of increasing 
workload. However, if there was a medical contraindication, the exercise testing was interrupted. The highest $20 \mathrm{~s}$ value for $\dot{V} \mathrm{O}_{2 \text { peak }}(1 / \mathrm{min})$ attained in the last min was used in the analysis and is termed 'CRF' from here on.

Defining CRF improvement CRF improvement was defined as having a change in $\dot{V} \mathrm{O}_{2 \text { peak }}(\mathrm{l} / \mathrm{min}) \geq 5.0 \%$ from baseline to follow-up. This cut-off point was chosen based on the technical error of measurement (TEM) of $\dot{V} \mathrm{O}_{2 \text { peak }}$ using the Cosmed $\mathrm{K} 4$, which is $\sim 4.5 \%$ [26]; this was also the cut-off point used by Pandey et al in a similar analysis with a type 2 diabetes population [14]. Any value greater than the TEM is more likely to be a true biological response to exercise training rather than a result of the typical error variation present in the measurement technique and the day-to-day biological variability present in the individual [27].

Laboratory measurements Participants underwent biochemical assessments, including the analysis of $\mathrm{HbA}_{1 \mathrm{c}}$. Blood samples were collected from an indwelling catheter and drawn into chilled, heparinised tubes and centrifuged rapidly to avoid glycolysis. $\mathrm{HbA}_{1 \mathrm{c}}$ was analysed by immunoassay (Premier $\mathrm{Hb} 9210 \mathrm{HbA}_{1 \mathrm{c}}$ Analyzer; Trinity Biotech, Wicklow, Ireland).

Physical activity Physical activity was assessed using an accelerometer (ActiGraph, GT3X+ model; Fort Walton Beach, FL, USA). Participants were asked to wear the accelerometer on the right hip, close to the iliac crest, for 7 consecutive days. The device activation, download and processing were performed using the software, Actilife (v.6.9.1; ActiGraph). The devices were activated on the first day, in the morning, and data were recorded using the raw mode with a $100 \mathrm{~Hz}$ frequency, and posteriorly downloaded into $15 \mathrm{~s}$ epochs. Apart from actual non-wear time (i.e. when the device was removed during sleep and water activities), periods of at least 60 consecutive min of zero activity intensity counts were considered as non-wear time. A valid day was considered as having $600 \mathrm{~min}$ or more of wear time. Participants with at least 4 valid days (including 1 weekend day) were included in the analyses. All minutes for which the accelerometer counts were below $100 \mathrm{cpm}$ were defined as sedentary time. Accelerometer counts $\geq 100 \mathrm{cpm}$ and $<2020 \mathrm{cpm}$ were classified as low-intensity physical activity (LIPA). Accelerometer counts $\geq 2020 \mathrm{cpm}$ were classified as moderate-to-vigorous physical activity (MVPA) [28].

Statistical analysis Descriptive statistics, including measures of central tendency (mean) and variability (SD) for normally distributed variables, and median (interquartile range) for skewed variables, were used to describe the baseline descriptive characteristics of the control group and those in the exercise groups who had improved (CRF responders) and did not have improved (CRF non-responders) CRF. To compare differences in descriptive characteristics and changes in physical activity between the three groups, a one-way ANOVA with Bonferroni adjustment for multiple comparisons or the non-parametric Kruskal-Wallis test was used to compare differences in continuous measures, and a $\chi^{2}$ test or Fisher's exact test was used for categorical variables.

Generalised estimating equations were used to model outcomes using sex and total number of training sessions completed as covariates. Since arterial stiffness is dependent on arterial pressure [29], per cent change in MAP from baseline to the end of the study was included as a covariate in all regression models assessing differences in arterial stiffness and structure indices. Further adjustment for baseline MVPA and the exercise intervention group (i.e. non-exercise control, HIIT or MCT) were also performed given the significant difference between CRF responder groups at baseline. A least significant difference post hoc test was used to estimate the between- and within-group effects with regard to haemodynamic and vascular outcomes. A linear distribution for the response was assumed and an autoregressive correlation matrix was set to the data.

A $p$ value of $<0.05$ was considered statistically significant. Data analyses were performed using IBM SPSS Statistics version 22.0 (SPSS, Chicago, IL, USA) and STATA version 13.1 (StataCorp, College Station, TX, USA).

\section{Results}

Of the 41 individuals with type 2 diabetes who engaged in 1 year of either MCT or HIIT combined with RT, 14 had clinically meaningful improvements in CRF (mean $\Delta \dot{V} \mathrm{O}_{2 \text { peak }}, 0.24 \mathrm{l} / \mathrm{min}$ ), whereas 27 participants did not have improvements in CRF (mean $\Delta \dot{V} \mathrm{O}_{2 \text { peak }},-0.16 \mathrm{l} / \mathrm{min}$ ) following exercise training. There were no significant difference in the number of training sessions that were attended by those in whom CRF was improved and not improved $(p=0.12)$. Baseline descriptive characteristics of the participants are presented in Table 1. At baseline there were no differences $(p>0.05)$ between the control group, those in whom CRF was improved and those in whom CRF was not improved, with the exception of MVPA, which was higher $(p<0.05)$ in CRF non-responders compared with the control group $(p<0.05)$, and the exercise intervention group category, with more of the CRF non-responders being in the HIIT group compared with CRF responders $(p<0.05)$.

Time in LIPA and MVPA were not significantly different between the CRF responders ( $\triangle$ LIPA, $6.9 \pm 57.9 \mathrm{~min}$; $\triangle \mathrm{MVPA}, 1.1 \pm 18.0 \mathrm{~min}$ ), non-responders ( $\triangle \mathrm{LIPA},-11.1 \pm$ $40.8 \mathrm{~min} ; \triangle \mathrm{MVPA},-2.8 \pm 22.9 \mathrm{~min}$ ) and control participants ( $\Delta$ LIPA, $0.2 \pm 70.5 \mathrm{~min} ; \Delta \mathrm{MVPA},-0.1 \pm 12.6 \mathrm{~min}$ ). 
Table 1 Baseline descriptive characteristics of individuals in the control group, and exercise groups with either improved or unimproved CRF following 1 year of exercise training

\begin{tabular}{|c|c|c|c|c|}
\hline Characteristic & Control $(n=22)$ & CRF non-responders $(n=27)$ & CRF responders $(n=14)$ & $p$ value $^{\mathrm{a}}$ \\
\hline Age, years & $60.8 \pm 7.5$ & $59.9 \pm 9.2$ & $56.6 \pm 4.9$ & 0.29 \\
\hline $\operatorname{Sex}(\mathrm{F}[n]: \mathrm{M}[n])$ & $11: 11$ & $11: 16$ & $6: 8$ & 0.81 \\
\hline Weight (kg) & $85.9 \pm 15.7$ & $81.8 \pm 13.1$ & $83.3 \pm 19.3$ & 0.65 \\
\hline Height (cm) & $164.5 \pm 9.5$ & $164.2 \pm 8.7$ & $165.3 \pm 7.6$ & 0.93 \\
\hline BMI $\left(\mathrm{kg} / \mathrm{m}^{2}\right)$ & $31.7 \pm 4.7$ & $30.5 \pm 5.2$ & $30.4 \pm 6.1$ & 0.69 \\
\hline Training sessions completed $(n)$ & NA & $95(31)$ & $110(16)$ & 0.12 \\
\hline Training sessions completed $(\%)$ & NA & $72.0(23.8)$ & $83.0(12.4)$ & 0.12 \\
\hline $\operatorname{MCT}(n): \operatorname{HIIT}(n)$ & NA & $11: 16$ & $11: 3^{\dagger}$ & 0.02 \\
\hline Time from DM dx, years & $5.9 \pm 5.4$ & $7.6 \pm 4.7$ & $7.9 \pm 5.5$ & 0.41 \\
\hline Baseline sedentary time (min/day) & $599.1(138.1)$ & $594.4(84.7)$ & $590.9(131.3)$ & 0.99 \\
\hline Baseline MVPA (min/day) & $15.9(21.0)$ & $31.8(34.9)$ & $35.0(41.3)^{*}$ & 0.02 \\
\hline Baseline LIPA (min/day) & $191.7 \pm 75.1$ & $201.4 \pm 55.2$ & $227.1 \pm 97.2$ & 0.36 \\
\hline Baseline $\mathrm{HbA}_{1 \mathrm{c}}(\mathrm{mmol} / \mathrm{mol})$ & $48.1(16.4)$ & $47.6(18.6)$ & $49.2(25.1)$ & 0.78 \\
\hline Baseline $\mathrm{HbA}_{1 \mathrm{c}}(\%)$ & $6.6(1.5)$ & $6.5(1.7)$ & $6.7(2.3)$ & 0.78 \\
\hline Baseline fasting glucose (mmol/l) & $7.7(3.1)$ & $7.9(3.5)$ & $8.0(6.4)$ & 0.21 \\
\hline Baseline $\dot{V} \mathrm{O}_{2 \text { peak }}(1 / \mathrm{min})$ & $2.14 \pm 0.59$ & $2.13 \pm 0.50$ & $1.98 \pm 0.29$ & 0.60 \\
\hline Baseline TTE (min) & $6.8 \pm 2.6$ & $7.6 \pm 2.3$ & $7.7 \pm 1.7$ & 0.36 \\
\hline Baseline resting HR (bpm) & $67.2 \pm 10.5$ & $66.6 \pm 9.3$ & $69.0 \pm 11.6$ & 0.77 \\
\hline Baseline total fat (kg) & $29.9 \pm 6.8$ & $27.7 \pm 7.6$ & $28.5 \pm 11.2$ & 0.64 \\
\hline Baseline MAP (mmHg) & $98.9 \pm 8.3$ & $101.8 \pm 9.6$ & $99.6 \pm 10.6$ & 0.54 \\
\hline
\end{tabular}

Data are presented as mean $\pm \mathrm{SD}$ or median (interquartile range), unless otherwise indicated

${ }^{a} p$ value for differences across all groups based on a one-way ANOVA with Bonferroni adjustment for multiple comparisons or the non-parametric Kruskal-Wallis test for continuous measures, and a $\chi^{2}$ test or Fisher's exact test for categorical variables

${ }^{*} p<0.05$ vs control, non-parametric Kruskal-Wallis test for continuous measures

${ }^{\dagger} p<0.05$ vs CRF non-responders, Fisher's exact test for categorical variables

bpm, beats per min; DM dx, diabetes diagnosis; F, female; HR, heart rate; M, male; NA, not applicable; TTE, time to exhaustion

Table 2 presents the between- and within-group changes for haemodynamic measures, and local and regional indices of early pathological changes in arterial structure and stiffness, after adjusting for sex, total number of training sessions completed, and exercise intervention group. Arterial structure and stiffness indices were additionally adjusted for per cent change in MAP. Regardless of whether CRF improved, those in the exercise groups had either decreased (CRF nonresponders: $-0.03 \mathrm{~mm}$ ) or unchanged (CRF responders: $0.001 \mathrm{~mm}$ ) carotid IMT compared with the control group $(+0.04 \mathrm{~mm})$, following the 1 year intervention. Improvements $(p<0.05)$ were also observed in peripheral arterial stiffness in exercisers, regardless of improvements in CRF, as lower-limb PWV was decreased vs the control group (CRF responders: $-0.38 \mathrm{~m} / \mathrm{s}$; CRF non-responders: $-0.40 \mathrm{~m} / \mathrm{s}$; control: $+1.31 \mathrm{~m} / \mathrm{s}$ ). However, only the CRF responders had significantly decreased PWV of the upper limb compared with control participants (CRF responders: $-1.29 \mathrm{~m} / \mathrm{s}$; control: $+0.12 \mathrm{~m} / \mathrm{s}$ ). As for central stiffness, only the CRF non-responders had significant changes in carotid distensibility coefficient $(p<0.05)$ compared with the control group. Despite changes in aortic PWV not differing from the control group $(p>0.05)$, changes in aortic PWV differed $(p<0.05)$ between CRF responders and non-responders, with non-responders having increased aortic PWV (CRF non-responders: $1.62 \mathrm{~m} / \mathrm{s}$ ), while the responders had decreased levels (CRF responders: $-0.68 \mathrm{~m} / \mathrm{s}$ ) following 1 year of exercise training. No other interaction effects between individuals in the exercise groups who had improved or who did not have improved CRF vs individuals in the control group were found for the remaining vascular stiffness or haemodynamic measures $(p>0.05)$. Further adjustment for baseline MVPA did not alter the results (data not shown).

Figure 1 displays the individual per cent changes in local and regional indices of early pathological changes in indices of arterial structure and stiffness in the control participants and individuals in the exercise groups who either had increased or not improved post-trial CRF. Even among the participants in the exercise groups with and without improvements in CRF, there was a wide range of interindividual variability in vascular adaptations to exercise training. Overall, compared with the control group, those in the exercise groups had 


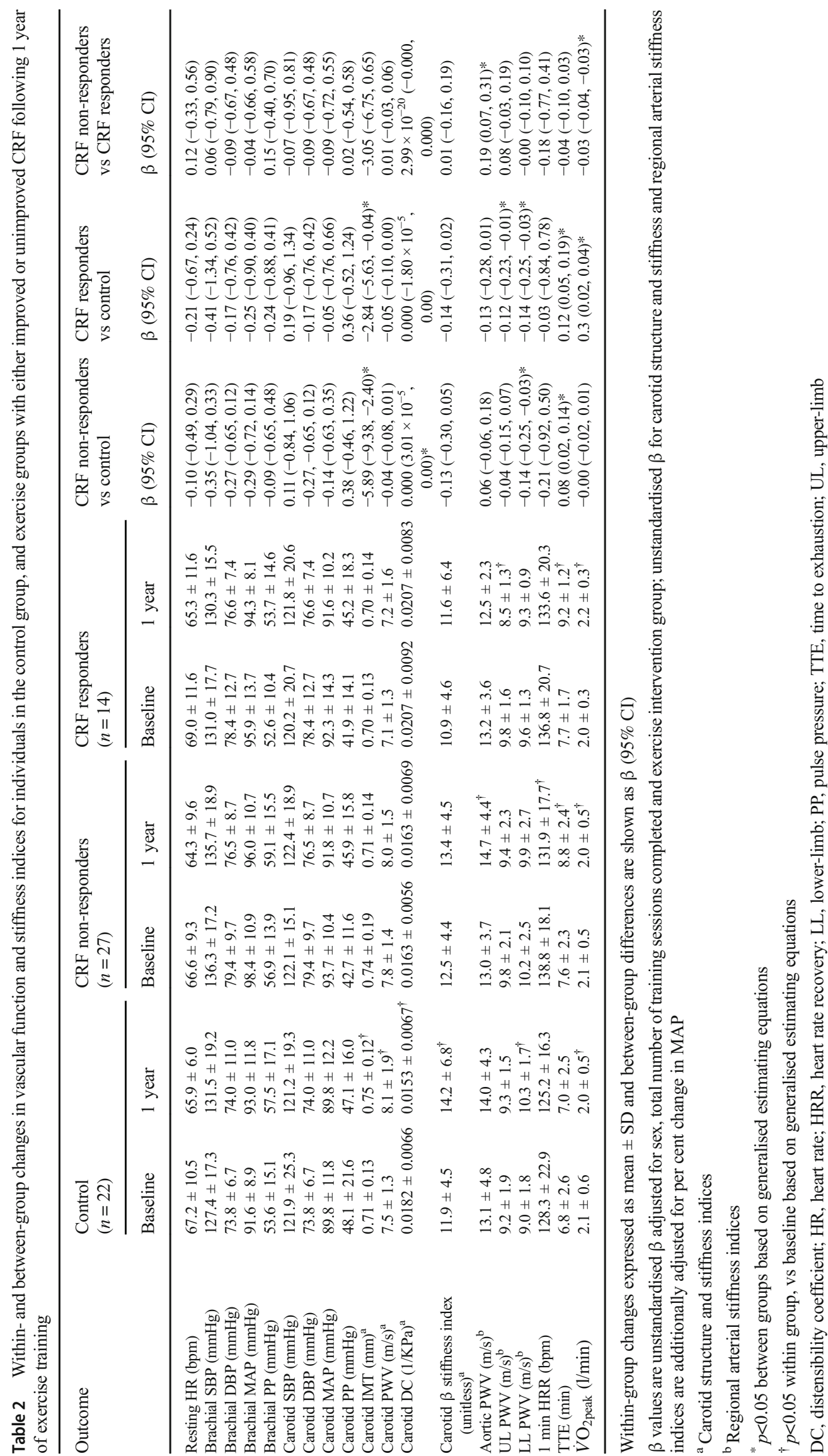



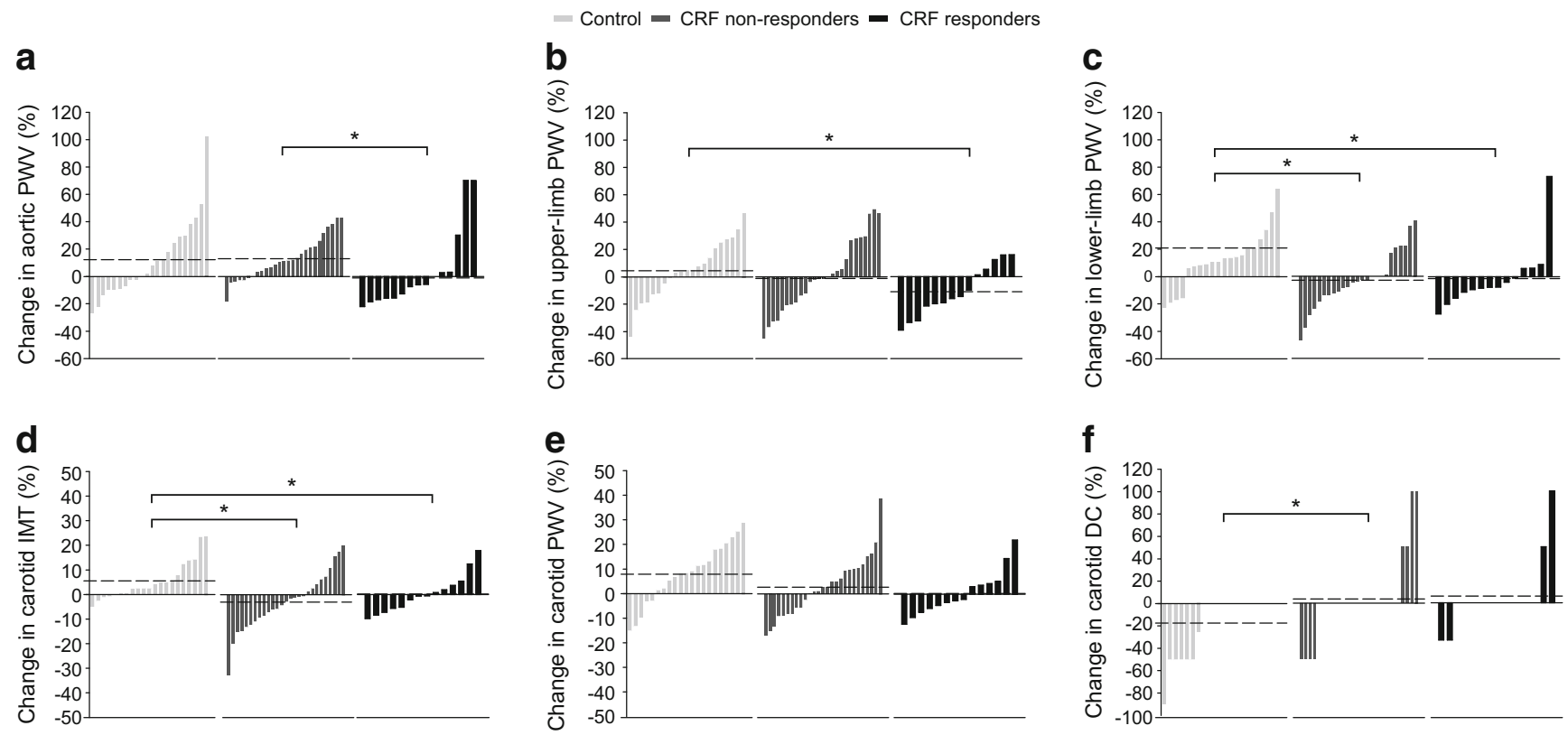

Fig. 1 Individual per cent changes in (a) aortic PWV, (b) upper-limb PWV, (c) lower-limb PWV, (d) carotid IMT, (e) carotid PWV and (f) carotid distensibility coefficient (DC). * $p<0.05$ vs control after adjustment for sex, total number of training sessions completed and per cent change in MAP

improvements $(p<0.05)$ in lower-limb PWV and carotid IMT (Fig. 1c, d), regardless of whether they had concurrent improvements in CRF.

\section{Discussion}

The aim of this study was to assess whether improvements in vascular function were independent of improvements in CRF, based on $\Delta \dot{V} \mathrm{O}_{2 \text { peak }}$, following 1 year of exercise training in individuals with type 2 diabetes. This is the first study to show that a long-term exercise intervention significantly decreased carotid IMT and lower-limb arterial stiffness in participants with type 2 diabetes, regardless of improvements in CRF. These findings are important given that vascular complications are common in individuals with type 2 diabetes and that these vascular alterations are a main mechanism connecting diabetes to increased risk of cardiovascular morbidity and mortality $[1,30]$. Hence, our findings suggest that exerciseinduced benefits in terms of vascular health may disrupt the close link between diabetes and CVD. In addition, individuals with type 2 diabetes are known to have CRF impairments independent of physical activity levels, obesity and presence of CVD [19], making them more likely to not experience changes in CRF following exercise, as compared with adults without type 2 diabetes [14, 31]. Thus, although exercise training-induced improvements in CRF have been correlated with decreases in CVD risk factors [10], this is the first study to show that the absence of an increase in CRF after exercise training does not preclude the possibility of beneficial vascular adaptations in individuals with type 2 diabetes.
In the present study, an interaction between CRF responders and non-responders was found in aortic PWV, such that individuals who had improved CRF with exercise had more favourable changes in aortic PWV. Strong associations between improvements in aortic vascular stiffness and reduced risk of cardiovascular and all-cause mortality have been previously reported [32]. However, using a central surrogate of PWV measured through carotid PWV, no differences between the exercise and control groups were found. Although carotid and aortic PWV are both classified as elastic and valid surrogate measures of central stiffness [2, 3], the ultrastructure of the carotid artery is more like the abdominal aorta than the ascending aorta. In addition, discrepancies between changes in carotid and aortic artery PWV following an intervention could also result from differences in measurement techniques: aortic PWV is determined manually by measuring the distance between the carotid and femoral sites, whereas carotid PWV is determined cross-sectionally from local changes in pressure and artery diameter due to the short vessel path $(10-15 \mathrm{~cm})$ and subsequent short time lag (5$15 \mathrm{~ms}$ ) of the carotid artery, making manual measurement difficult [33]. Nevertheless, compared with the control group, decreases in PWV of the lower limb of exercisers with and without improvements in CRF were observed suggesting that exercise training may have a larger effect on peripheral rather than central stiffness, which may be attributable to the mechanical compression of the arteries due to muscular contraction during exercise [34]. In line with our findings, a recent meta-analysis of the impact of exercise on both central and peripheral arterial stiffness in adults, observed that exercise training had less of an effect on central arterial stiffness 
compared with peripheral measures [35]. Even though aortic PWV is the only segment of the arteries associated with decreased CVD risk, changes in lower-limb PWV following exercise might induce local adaptations and have implications on other systems, such as glucose transport and insulin signalling, in the muscle [36].

Beyond PWV, the distensibility coefficient, measured through ultrasound of the carotid artery, is also an indicator of vascular stiffness, which tends to deteriorate with ageing and type 2 diabetes $[37,38]$. We found that, regardless of CRF improvement, 1 year of exercise training was able to halt the natural age- and disease-related deterioration in carotid distensibility vs baseline, such that decreases in carotid distensibility coefficient were only observed in control participants.

Exercise training has also been shown to improve indices of local vascular structure [6]. In accordance with previous findings, we also observed favourable effects of exercise training on carotid IMT independent of improvements in CRF. Given that for every $0.1 \mathrm{~mm}$ increase in carotid IMT, the risk of having a myocardial infarction increases by $\sim 10 \%$ [4], our finding of a decrease in carotid IMT by $0.03 \mathrm{~mm}$ in the CRF non-responders and maintenance of carotid IMT in responders has important clinical implications in terms of decreasing cardiovascular events, which can occur regardless of having changes in CRF. In addition, it is important to note that carotid IMT is strongly linked to age and has been estimated to increase by $\sim 0.010 \mathrm{~mm}$ per year in men and women [39]. Hence, our finding of a decrease and/or maintenance of carotid IMT after 1 year is even more relevant as it indicates that regardless of CRF improvements, long-term exercise can slow down the age-related increases in IMT, or even promote a decrease in carotid IMT.

Currently, we have only fragments of insight regarding the mechanisms involved in vascular remodelling following long-term exercise training regimes. Teasing apart the contribution of CRF on the vascular system [40] may provide key information for tailored interventions aiming for the prevention of arterial stiffening and related cardiovascular complications. In fact, most of the vascular benefits observed with exercise are thought to be mediated by the increased exercise-induced blood flow, which is responsible for increasing endothelial shear stress [11]. The force exerted by the blood flow on the arterial wall (i.e. shear stress) stimulates the production of nitric oxide, a potent vasodilator that adjusts the shear stress to which the artery is subjected to and plays a key role in repairing damaged endothelial cells, leading to an overall improvement in vascular health with medium-to-long-term implications on vascular structure and function [11]. This shear stress and pulsatile stretching of the arteries is also believed to aid in the breakdown of diabetes-associated collagen cross-links, resulting in decreased arterial stiffness [11]. Exercise-related improvements in vascular health may also be influenced by exercise-induced changes in metabolic disease risk factors, including hypertension, dyslipidaemia, inflammation and insulin resistance [41]. However, although CRF is associated with a favourable metabolic profile [40], several reports suggest that the exercise-induced metabolic improvements, which in turn improve vascular health, are independent of changes in CRF [18]. For instance, improvements in metabolic parameters, such as $\mathrm{HbA}_{1 \mathrm{c}}$ and measures of adiposity, have been observed in individuals with type 2 diabetes, irrespective of improvements in CRF, following a 9 month exercise intervention [14]. Additionally, it has been suggested that the high interindividual variability in response to exercise can be partially due to alterations in non-exercise physical activity (NEPA), which is known to be affected by the intensity and duration of exercise training sessions [42]. However, we did not observe any differences in LIPA and MVPA levels between control participants and those in the exercise intervention, regardless of improvements in CRF, suggesting that improvements in vascular outcomes in the present study were not a consequence of higher NEPA.

Understanding the factors that lead to an increase in CRF in some but not all individuals undertaking an exercise intervention remains a source of considerable discussion [15]. Currently, there is no consensus on how to best quantify individual variability and categorise responders and nonresponders to an exercise intervention. For instance, previous investigations have used various approaches to classify a CRF non-responder, including: a $\leq 0 \mathrm{l} / \mathrm{min}$ change in CRF [43, 44]; a non-clinically significant $<5 \%$ change in CRF [14]; a withinparticipant $\mathrm{CV}$ of $5.6 \%$ from the literature [45]; arbitrary cutoff points of CRF $\leq 0 \mathrm{ml} \mathrm{kg} \mathrm{min}^{-1}, \leq 1.75 \mathrm{ml} \mathrm{kg}^{-1} \mathrm{~min}^{-1}$ and $\leq 3.5 \mathrm{ml} \mathrm{kg}^{-1} \mathrm{~min}^{-1}$ [46]; and a change in CRF $\leq \mathrm{TEM}[31,47$ ]. Nonetheless, all of these investigations have focused on the use of $\dot{V} \mathrm{O}_{2}$ (i.e. either maximal or peak) as the indicator of CRF. However, there are other indices of CRF that may provide additional information on the effectiveness of exercise interventions. Our results show that despite the absence of CRF improvements based on $\dot{V} \mathrm{O}_{2 \text { peak }}$ in the non-responder group, both CRF responders and non-responders had increased time to exhaustion during maximal CRF exercise testing at 1 year vs baseline. It is possible that the increases in the time to exhaustion observed in the non-responder group were due to improvements in walking performance and other efficiency factors as a result of the exercise intervention, which may potentially contribute to more favourable effects on the economy of motion without leading to a concurrent increase in the $\dot{V} \mathrm{O}_{2 \text { peak }}$ [48]. Moreover, previous investigations have suggested that there is substantial variability in the time to exercise exhaustion, with increases in time to 
exhaustion being independent of changes in $\dot{V} \mathrm{O}_{2 \max }$ over a series of repeated exercise tests $[49,50]$.

This study is not without limitations. Although our sample size was relatively large considering the length of the exercise intervention, the small number of participants within each group (i.e. control, CRF non-responders and CRF responders) could have affected the power to detect significant changes between groups.

In conclusion, 1 year of exercise training was able to slow the progression of the natural age- and disease-related deterioration of vascular health in individuals with type 2 diabetes, with significant improvements being observed in carotid IMT and lower limb arterial stiffness vs a non-exercise control group, regardless of changes in CRF. Thus, a lack of improvement in CRF following exercise in individuals with type 2 diabetes does not necessarily entail a lack of improvement in vascular health. From a clinical standpoint, when assessing the successfulness of an exercise intervention in individuals with type 2 diabetes, practitioners should not overlook the impact of exercise training on other health indicators by simply focusing on CRF, as other favourable health benefits are still obtainable with exercise in this patient population despite lack of improvements in fitness.

Acknowledgements The authors are grateful to all participants for their time and effort.

\section{Data availability Data are available on request from the authors.}

Funding This work was supported by fellowships from the Portuguese Foundation for Science and Technology (grant to JPM: SFRH/BD/ 85742/2012; grant to PBJ: SFRH/BPD/115977/2016). This work is also financed by a national grant through the Fundação para a Ciência e Tecnologia (FCT) within the unit I\&D 472 (UID/DTP/00447/2019).

Duality of interest The authors declare that there is no duality of interest associated with this manuscript.

Contribution statement LBS and JPM contributed to the conception and design of the study. JPM, PBJ and XM were responsible for data collection and acquisition. MHR was responsible for data analysis and interpretation. MHR drafted the manuscript. LBS, JPM, PBJ and XM contributed to reviewing and editing the manuscript. LBS and MHR gave approval of the final version of the manuscript and take responsibility for the integrity of the data and the accuracy of the data analysis.

\section{References}

1. Yahagi K, Kolodgie FD, Lutter C et al (2017) Pathology of human coronary and carotid artery atherosclerosis and vascular calcification in diabetes mellitus. Arterioscler Thromb Vasc Biol 37(2):191204. https://doi.org/10.1161/atvbaha.116.306256

2. Cardoso CR, Salles GF (2016) Aortic stiffness as a surrogate endpoint to micro- and macrovascular complications in patients with type 2 diabetes. Int J Mol Sci 17(12):2044. https://doi.org/ 10.3390/ijms 17122044
3. Vlachopoulos C, Aznaouridis K, Stefanadis C (2010) Prediction of cardiovascular events and all-cause mortality with arterial stiffness: a systematic review and meta-analysis. J Am Coll Cardiol 55(13): 1318-1327. https://doi.org/10.1016/j.jacc.2009.10.061

4. Lorenz MW, Markus HS, Bots ML, Rosvall M, Sitzer M (2007) Prediction of clinical cardiovascular events with carotid intimamedia thickness: a systematic review and meta-analysis. Circulation 115(4):459-467. https://doi.org/10.1161/ CIRCULATIONAHA.106.628875

5. Madden KM, Lockhart C, Cuff D, Potter TF, Meneilly GS (2013) Aerobic training in older adults with type 2 diabetes and vasodepressive carotid sinus hypersensitivity. Aging Clin Exp Res 25(6):651-657. https://doi.org/10.1007/s40520-013-0160-4

6. Byrkjeland R, Stensaeth KH, Anderssen S et al (2016) Effects of exercise training on carotid intima-media thickness in patients with type 2 diabetes and coronary artery disease. Influence of carotid plaques. Cardiovasc Diabetol 15(1):13. https://doi.org/10.1186/ s12933-016-0336-2

7. Green DJ, Hopman MT, Padilla J, Laughlin MH, Thijssen DH (2017) Vascular adaptation to exercise in humans: role of hemodynamic stimuli. Physiol Rev 97(2):495-528. https://doi.org/10.1152/ physrev.00014.2016

8. Magalhães JP, Melo X, Correia IR et al (2019) Effects of combined training with different intensities on vascular health in patients with type 2 diabetes: a 1-year randomized controlled trial. Cardiovasc Diabetol 18(1):34. https://doi.org/10.1186/s12933-019-0840-2

9. Joyner MJ, Green DJ (2009) Exercise protects the cardiovascular system: effects beyond traditional risk factors. J Physiol 587(23): 5551-5558. https://doi.org/10.1113/jphysiol.2009.179432

10. Hamer M, O'Donovan G (2010) Cardiorespiratory fitness and metabolic risk factors in obesity. Curr Opin Lipidol 21(1):1-7. https://doi.org/10.1097/MOL.0b013e328331dd21

11. Zieman SJ, Melenovsky V, Kass DA (2005) Mechanisms, pathophysiology, and therapy of arterial stiffness. Arterioscler Thromb Vasc Biol 25(5):932-943. https://doi.org/10.1161/01.ATV. 0000160548.78317 .29

12. Jae SY, Heffernan K, Fernhall B, Choi YH (2012) Cardiorespiratory fitness and carotid artery intima media thickness in men with type 2 diabetes. J Phys Act Health 9(4):549-553. https://doi.org/10.1123/ jpah.9.4.549

13. Donley DA, Fournier SB, Reger BL et al (2014) Aerobic exercise training reduces arterial stiffness in metabolic syndrome. J Appl Physiol 116(11):1396-1404. https://doi.org/10.1152/japplphysiol. 00151.2014

14. Pandey A, Swift DL, McGuire DK et al (2015) Metabolic effects of exercise training among fitness-nonresponsive patients with type 2 diabetes: the HART-D Study. Diabetes Care 38(8):1494-1501. https://doi.org/10.2337/dc14-2378

15. Ross R, Goodpaster BH, Koch LG et al (2019) Precision exercise medicine: understanding exercise response variability. Br J Sports Med 53(18):1141-1153. https://doi.org/10.1136/bjsports-2018100328

16. Laye MJ, Nielsen MB, Hansen LS, Knudsen T, Pedersen BK (2015) Physical activity enhances metabolic fitness independently of cardiorespiratory fitness in marathon runners. Dis Markers 2015: 806418. https://doi.org/10.1155/2015/806418

17. Kraus WE, Houmard JA, Duscha BD et al (2002) Effects of the amount and intensity of exercise on plasma lipoproteins. N Engl J Med 347(19):1483-1492. https://doi.org/10.1056/NEJMoa020194

18. Hartman YAW, Hopman MTE, Schreuder TH et al (2018) Improvements in fitness are not obligatory for exercise traininginduced improvements in CV risk factors. Phys Rep 6(4):e13595. https://doi.org/10.14814/phy2.13595

19. Wahl MP, Scalzo RL, Regensteiner JG, Reusch JEB (2018) Mechanisms of aerobic exercise impairment in diabetes: a narrative 
review. Front Endocrinol 9:181. https://doi.org/10.3389/fendo. 2018.00181

20. Magalhaes JP, Judice PB, Ribeiro R et al (2019) Effectiveness of high-intensity interval training combined with resistance training versus continuous moderate-intensity training combined with resistance training in patients with type 2 diabetes: a one-year randomized controlled trial. Diabetes Obes Metab 21(3):550-559. https:// doi.org/10.1111/dom.13551

21. Diabetes Care (2017) Standards of medical care in diabetes-2017: summary of revisions. Diabetes Care 40(Suppl 1):S4-S5. https:// doi.org/10.2337/dc17-S003

22. U.S. Department of Health and Human Services (2008) 2008 Physical Acivity Guidelines for Americans, 2nd edn. U.S. Department of Health and Human Services, Washington, DC

23. Lohman TG, Roche AF, Martorell R (1988) Anthropometric standardization reference manual. Human Kinetics Publishers, Champaign, IL

24. Hoeks AP, Willekes C, Boutouyrie P, Brands PJ, Willigers JM, Reneman RS (1997) Automated detection of local artery wall thickness based on M-line signal processing. Ultrasound Med Biol 23(7):1017-1023. https://doi.org/10.1016/S0301-5629(97)00119-1

25. Bruce RA (1971) Exercise testing of patients with coronary heart disease. Principles and normal standards for evaluation. Ann Clin Res 3(6):323-332

26. Duffield R, Dawson B, Pinnington HC, Wong P (2004) Accuracy and reliability of a Cosmed $\mathrm{K} 4 \mathrm{~b} 2$ portable gas analysis system. J Sci Med Sport 7(1):11-22. https://doi.org/10.1016/s1440-2440(04) 80039-2

27. Harris EF, Smith RN (2009) Accounting for measurement error: a critical but often overlooked process. Arch Oral Biol 54(Suppl 1): S107-S117. https://doi.org/10.1016/j.archoralbio.2008.04.010

28. Troiano RP, Berrigan D, Dodd KW, Masse LC, Tilert T, McDowell M (2008) Physical activity in the United States measured by accelerometer. Med Sci Sports Exerc 40(1):181-188. https://doi.org/10. 1249/mss.0b013e31815a51b3

29. Melo X, Fernhall B, Santos DA et al (2016) The acute effect of maximal exercise on central and peripheral arterial stiffness indices and hemodynamics in children and adults. Appl Physiol Nutr Metab 41(3):266-276. https://doi.org/10.1139/apnm-2015-0204

30. Stehouwer CD, Henry RM, Ferreira I (2008) Arterial stiffness in diabetes and the metabolic syndrome: a pathway to cardiovascular disease. Diabetologia 51(4):527-539. https://doi.org/10.1007/ s00125-007-0918-3

31. Montero D, Lundby C (2017) Refuting the myth of non-response to exercise training: 'non-responders' do respond to higher dose of training. J Physiol 595(11):3377-3387. https://doi.org/10.1113/ JP273480

32. Guerin AP, Blacher J, Pannier B, Marchais SJ, Safar ME, London GM (2001) Impact of aortic stiffness attenuation on survival of patients in end-stage renal failure. Circulation 103(7):987-992. https://doi.org/10.1161/01.cir.103.7.987

33. Paini A, Boutouyrie P, Calvet D, Tropeano AI, Laloux B, Laurent S (2006) Carotid and aortic stiffness: determinants of discrepancies. Hypertension 47(3):371-376. https://doi.org/10.1161/01.HYP. 0000202052.25238 .68

34. Heffernan KS, Edwards DG, Rossow L, Jae SY, Fernhall B (2007) External mechanical compression reduces regional arterial stiffness. Eur J Appl Physiol 101(6):735-741. https://doi.org/10.1007/ s00421-007-0550-4

35. Ashor AW, Lara J, Siervo M, Celis-Morales C, Mathers JC (2014) Effects of exercise modalities on arterial stiffness and wave reflection: a systematic review and meta-analysis of randomized controlled trials. PLoS One 9(10):e110034. https://doi.org/10. 1371/journal.pone.0110034
36. Olver TD, Laughlin MH (2016) Endurance, interval sprint, and resistance exercise training: impact on microvascular dysfunction in type 2 diabetes. Am J Physiol Heart Circ Physiol 310(3):H337H350. https://doi.org/10.1152/ajpheart.00440.2015

37. Taddei S, Virdis A, Mattei P et al (1995) Aging and endothelial function in normotensive subjects and patients with essential hypertension. Circulation 91(7):1981-1987. https://doi.org/10.1161/01. cir.91.7.1981

38. Laurent S, Caviezel B, Beck L et al (1994) Carotid artery distensibility and distending pressure in hypertensive humans. Hypertension 23(6 Pt 2):878-883. https://doi.org/10.1161/01.hyp. 23.6.878

39. Touboul PJ (2002) Clinical impact of intima media measurement. Eur J Ultrasound 16(1-2):105-113. https://doi.org/10.1016/s09298266(02)00050-2

40. DeFina LF, Haskell WL, Willis BL et al (2015) Physical activity versus cardiorespiratory fitness: two (partly) distinct components of cardiovascular health? Prog Cardiovasc Dis 57(4):324-329. https:// doi.org/10.1016/j.pcad.2014.09.008

41. Salvi P (2012) Evaluation of the mechanical properties of large arteries. In: Salvi P (ed) Pulse waves. Springer, Milano, pp 17-44

42. Fedewa MV, Hathaway ED, Williams TD, Schmidt MD (2017) Effect of exercise training on non-exercise physical activity: a systematic review and meta-analysis of randomized controlled trials. Sports Med 47(6):1171-1182. https://doi.org/10.1007/ s40279-016-0649-Z

43. Sisson SB, Katzmarzyk PT, Earnest CP, Bouchard C, Blair SN, Church TS (2009) Volume of exercise and fitness nonresponse in sedentary, postmenopausal women. Med Sci Sports Exerc 41(3): 539-545. https://doi.org/10.1249/MSS.0b013e3181896c4e

44. Chmelo EA, Crotts CI, Newman JC et al (2015) Heterogeneity of physical function responses to exercise training in older adults. $\mathrm{J}$ Am Geriatr Soc 63(3):462-469. https://doi.org/10.1111/jgs. 13322

45. Scharhag-Rosenberger F, Walitzek S, Kindermann W, Meyer T (2012) Differences in adaptations to 1 year of aerobic endurance training: individual patterns of nonresponse. Scand J Med Sci Sports 22(1):113-118. https://doi.org/10.1111/j.1600-0838.2010. 01139.x

46. Johannsen NM, Swift DL, Lavie CJ, Earnest CP, Blair SN, Church TS (2013) Categorical analysis of the impact of aerobic and resistance exercise training, alone and in combination, on cardiorespiratory fitness levels in patients with type 2 diabetes: results from the HART-D study. Diabetes Care 36(10):3305-3312. https://doi.org/ $10.2337 / \mathrm{dc} 12-2194$

47. Ross R, de Lannoy L, Stotz PJ (2015) Separate effects of intensity and amount of exercise on interindividual cardiorespiratory fitness response. Mayo Clin Proc 90(11):1506-1514. https://doi.org/10. 1016/j.mayocp.2015.07.024

48. Beneke R, Meyer K (1997) Walking performance and economy in chronic heart failure patients pre and post exercise training. Eur $\mathrm{J}$ Appl Physiol Occup Physiol 75(3):246-251. https://doi.org/10. 1007/s004210050155

49. Gleser MA, Vogel JA (1971) Endurance exercise: effect of workrest schedules and repeated testing. J Appl Physiol 31(5):735-739. https://doi.org/10.1152/jappl.1971.31.5.735

50. McLellan TM, Cheung SS, Jacobs I (1995) Variability of time to exhaustion during submaximal exercise. Can J Appl Physiol 20(1): 39-51. https://doi.org/10.1139/h95-003

Publisher's note Springer Nature remains neutral with regard to jurisdictional claims in published maps and institutional affiliations. 